\title{
VALIDATING AN EEG-BASED FATIGUE DETECTION AND MITIGATION SYSTEM
}

\author{
Kuan-Chih Huang \\ Department of Electrical and Computer Engineering \\ National Chiao-Tung University \\ Hsinchu, Taiwan \\ Brain Research Center, University System of Taiwan \\ Hsinchu, Taiwan \\ Teng-Yi Huang, Chun-Hsiang Chuang, Jung-Tai King, Yu-Kai Wang \\ Brain Research Center, University System of Taiwan \\ Hsinchu, Taiwan \\ Chin-Teng Lin \\ Department of Electrical and Computer Engineering \\ National Chiao-Tung University \\ Hsinchu, Taiwan \\ Faculty of Engineering and Information Technology \\ University of Technology, Sydney \\ Sydney, Australia \\ Center for Advanced Neurological Engineering \\ Institute of Engineering in Medicine \\ University of California \\ San Diego, CA, USA \\ E-mail: ctlin@mail.nctu.edu.tw \\ Tzyy-Ping Jung \\ Center for Advanced Neurological Engineering \\ Institute of Engineering in Medicine \\ Swartz Center for Computational Neuroscience \\ Institute for Neural Computation \\ University of California \\ San Diego, CA, USA \\ E-mail: jung@sccn.ucsd.edu
}

\begin{abstract}
Research has indicated that fatigue is a critical factor in cognitive lapses because it negatively affects an individual's internal state, which is then manifested physiologically. This study explores neurophysiological changes, measured by electroencephalogram (EEG), due to fatigue. This study further demonstrates the feasibility of an on-line closed-loop EEG-based fatigue detection and mitigation system that detects physiological change and can thereby prevent fatigue-related cognitive lapses. More importantly, this work compares the efficacy of fatigue detection and mitigation between the EEG-based and a non-EEG-based random method. Twelve healthy subjects participated in a sustained-attention driving experiment. Each participant's EEG signal was monitored continuously and a warning was delivered in real time to participants once the EEG signature of fatigue was detected. Study results indicate suppression of the alpha- and theta-power of an occipital component and improved behavioral performance following a warning signal; these findings are in line with those in previous studies. However, study results also showed reduced warning efficacy (i.e., increased response times to lane deviations) accompanied by increased alpha-power due to the fluctuation of warnings over time. Furthermore, a comparison of EEG-based and non-EEG-based random approaches clearly demonstrated the necessity of adaptive fatigue mitigation systems,
\end{abstract}


based on a subject's cognitive level, to deliver warnings. Analytical results clearly demonstrate and validate the efficacy of this on-line closed-loop EEG-based fatigue detection and mitigation mechanism to identify cognitive lapses that may lead to catastrophic incidents in countless operational environments.

Keywords: EEG, Fatigue, Auditory Feedback, Brain Dynamics, Driving Safety.

\section{Introduction}

Fatigue is the induction of and generally accompanies drowsiness, a transitional state between wake and sleep. ${ }^{1}$ Further, fatigue accumulates gradually and associated with declines in attention, eventually reducing performance and efficiency. ${ }^{2}$ The resulting risk of injury or death is obvious when drivers are fatigued; that is, reaction time slows, situational awareness decreases, and judgment and vision are impaired. ${ }^{3,4}$ Driver fatigue is a major cause of crashes and negatively impacts road safety. Mechanisms that can identify fatigues are necessary to prevent fatigue-related accidents. ${ }^{5-8}$

Studies have deployed indicators to measure physiological changes, such as changes in blinking rate ${ }^{9}$ and heart rate, ${ }^{10}$ as a means of evaluating cognitive capability. A number of studies have also indicated that variation in an individual's behavioral performance accompanies significant changes in the electroencephalogram (EEG) power spectrum. ${ }^{11-26}$ Researchers have indicated that the EEG signal may be a very predictive and reliable indicator of alertness ${ }^{27}$ and can be used to develop devices to combat fatigue. ${ }^{19,25,28,29}$

Our recent study ${ }^{25}$ used an event-related lanekeeping task in a virtual environment and recorded brain dynamics via a noninvasive electroencephalogram (EEG) device. Subjects were instructed to steer a simulated car back to its original cruising position when it drifted into another lanes. Response time (RT), the time interval between deviation and response, was improved by auditory feedback delivered to drowsy subjects. Furthermore, the capacity of a subject to respond was correlated with variations in EEG power spectra.

Several studies have explored methods to mitigate fatigue-related cognitive lapses. Graham ${ }^{5}$ and Belz et al., ${ }^{30}$ for example, demonstrated that warning signals effectively improve the performance of drivers. Our previous studies ${ }^{25,} 31$ delivered an auditory warning signal at $1,750 \mathrm{~Hz}$ to drivers to restore performance that had been decreased by fatigue, and thereby reduced the magnitude of cognitive lapses. Furthermore, we have explored the effects of arousal feedback to reduce cognitive lapses in situations requiring attention ${ }^{25,32}$ and conduct an on-line lapse detection and mitigation system ${ }^{28}$ in real-world environments.

This study extends the previous work using an online closed-loop EEG-based fatigue detection system to predict driving fatigue based on EEG power spectra. This system validates the correlation between EEG and behavioral performance while driving before and after a warning delivered to drowsy subjects. Additionally, this study investigates that the efficacy of warning signals often declines over time. Furthermore, in order to validate the benefits of an EEG-based fatigue-mitigation system over those that do not use the EEG, this study compares the task performance obtained by the EEGbased and non-EEG-based random fatigue mitigation systems.

\section{Methods}

\subsection{Subjects}

In total, twelve healthy subjects ( 7 males and 5 females) aged 20-26 with normal hearing were recruited as paid volunteers for this virtual reality (VR)-based highway driving experiment. No subjects had neurological and psychological disorders and did not abuse drugs or alcohol. The subjects were instructed to get sufficient and regular sleep in the night before the experiment. No subject consumed alcohol or caffeine in the morning on the experiment day or had worked a night shift during the previous two months. The Institutional Review Board of the Taipei Veterans General Hospital approved the experimental protocol. All experiments were conducted in the early afternoon $(13: 30 \pm 1 \mathrm{~h})$ after lunch, when the circadian sleep rhythm becoming stronger.

All subjects were informed about experimental materials, features and the driving task process in advance. Subjects practiced driving for 5-10 min until they felt comfortable with experimental procedures. They were also asked to complete a questionnaire before and after the experiment. 


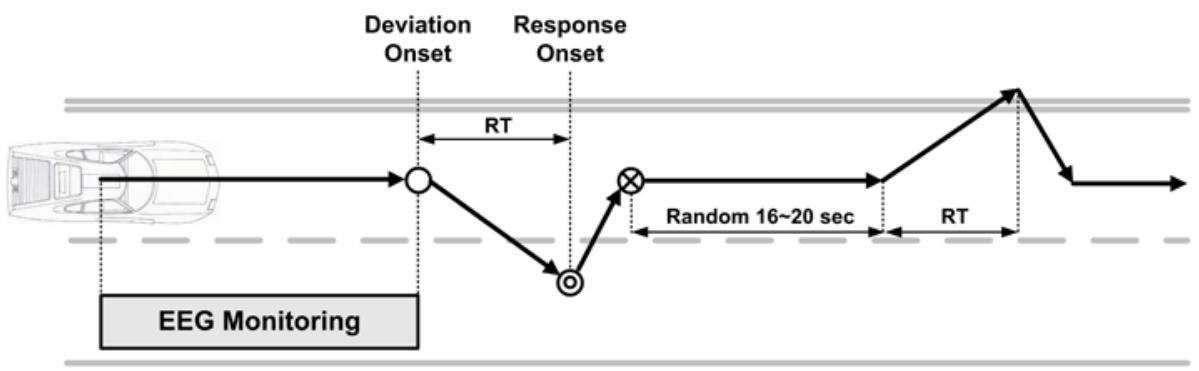

(a)

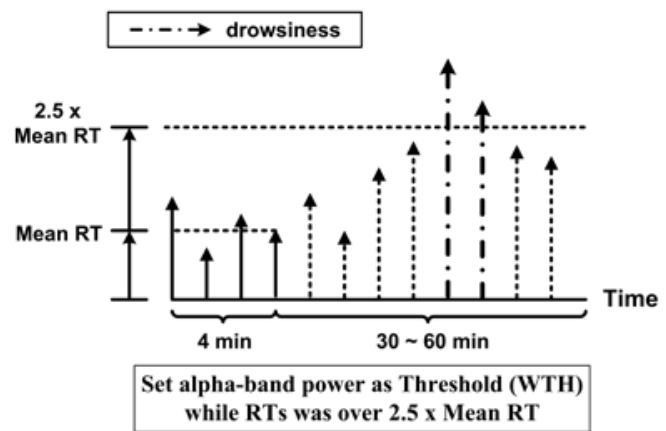

(b)

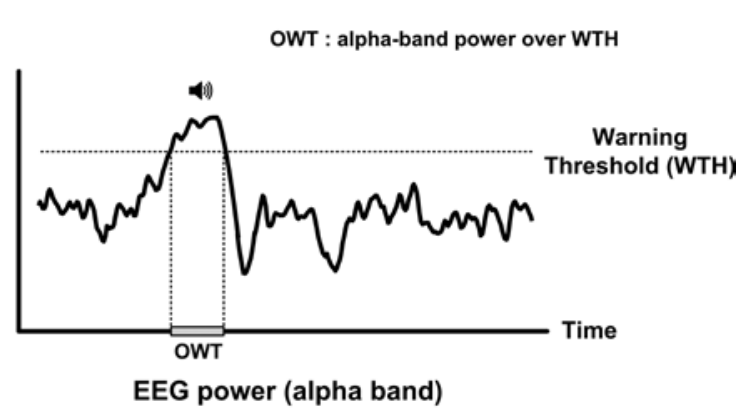

(c)

Fig. 1. Experimental paradigm. (a) Event-related lane-keeping tasks. The solid black arrows represent driving trajectory. The empty circle represents deviation onset. The double circle represents response onset. The circle with a cross represents end of response. Drivers' response time (RT) is the time interval from deviation onset (empty circle) to response onset (double circle). End of response (circle with a cross) means that drivers are steering car back into the original lane. The next deviation begins at 16-20 s after end of response [Adapted from Ref. 25]. (b) Setting a warning threshold while drivers became drowsy. The height of an arrow represents the response time of a single trial. The alpha-band power was recorded as the warning threshold (WTH) when a trial's RT was longer than 2.5 times the mean RT of trials within the first 4 min of the task during which the subject was asked to remain alert and attend to the tasks [Adapted from Ref. 25]. (c) Criteria for delivering auditory feedback to drivers during driving tasks. The subject's alpha-band power was calculated using the fast Fourier transform (FFT). A warning was delivered to drivers when their alpha-band power exceeded the WTH. The warning lasted until the driver's alpha-band power dipped below the WTH.

\subsection{Experimental equipment}

The VR-based highway driving experiments were performed in a simulator that mimicked a driving situation in a darkened sound-reduced room. The VR scene simulated driving at a constant speed of $100 \mathrm{~km} / \mathrm{h}$ on a four-lane divided highway with their car randomly drifting away from the center of the cruising lane to simulate driving on non-ideal road surfaces or with poor alignment. $^{24,25}$ In addition, a straight and monotonous road without traffic or stimuli was intended to simulate a long-time driving situation likely to induce drowsiness. The refresh rate of the highway scene was $60 \mathrm{~Hz}$. Scenes moved according to car displacement and steering.

During the experiment, the EEG activities were recorded by 30-channel scalp electrodes (Ag/AgCl electrodes) with a unipolar reference at the mastoid by the SynAmps system (Compumedics Ltd., VIC, Australia). The EEG electrodes were placed based on a modified international 10-20 system. Contact impedance between EEG electrodes and the cortex was calibrated to $<10 \mathrm{k} \Omega$. The EEG data were recorded with a 32-bit quantization level at a sampling rate of $500 \mathrm{~Hz}$ and preprocessed with a $50 \mathrm{~Hz}$ low-pass filter and a 0.5 $\mathrm{Hz}$ high-pass filter.

\subsection{Experimental paradigm}

An event-related lane-departure driving task (Fig. $1(\mathrm{a}))^{25}$ was used to assess objectively and quantitatively fluctuations in driving (behavioral) performance over long periods. Each driving session lasted 40-60 min, which was sufficient for subjects to experience fatigue. Furthermore, each experiment consisted of two sessions, a calibration session to determine a reasonable warning threshold (EEG power spectra in the alpha band) of 


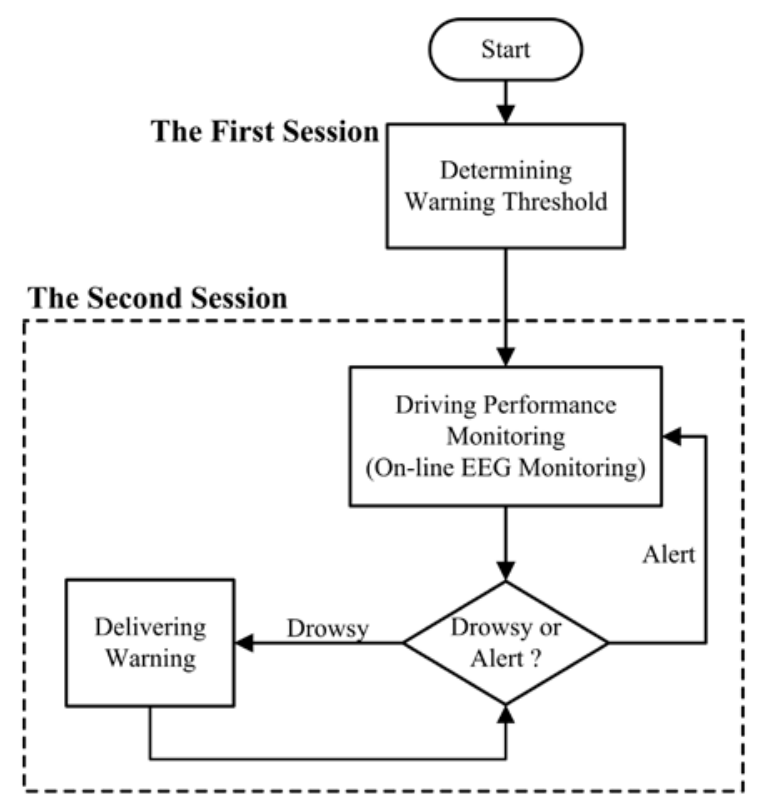

Fig. 2. The flowchart of the experimental protocol. The first session determined a rational warning threshold for each subject (cf. Fig. 1(b)). In the second session, a warning was delivered if the average alpha-band power exceeded the warning threshold (cf. Fig. 1(c)).

each subject (Fig. 1(b)), and an on-line session (Fig. 1(c)).

Fig. 1(a) shows the event-related lane-departure task. Lane-departure events were randomly introduced at 16-20 s intervals with the car drifting at a constant speed with equal probability toward the curb or into the opposite lane. ${ }^{25}$ Subjects were instructed to then steer the vehicle back toward the center of the original cruising lane as soon as possible. ${ }^{25}$ Before the experiment, subjects were instructed to remain as alert as possible. Experimental performance was monitored by a surveillance camera and vehicle trajectory was recorded. The times at which the car veered, when a response onset occurred, and when the response ended were recorded during each experiment. In Fig. 1, an empty circle represents an unexpected veering event, and is marked as “deviation onset”. Response onset was when subjects began to steer the car back toward the center of the cruising lane (double circle). The moment subjects stopped moving the steering wheel (circle with cross) was end of response. Response time (RT) was the time interval between the deviation onset and response onset.
The experiment had two sessions. Due to individual variability, suitable criteria for the warning needed to be determined from data acquired in the first calibration session (Fig. 2). The threshold of EEG alpha power was set according to a subject's behavioral performance in the session. ${ }^{25}$ Then, the second session used the spectral threshold to trigger a warning delivered to fatiguing subjects. Fig. 2 presents the flowchart of the closed-loop fatigue-monitoring system.

Fig. 1(b) shows the method used to set a suitable warning threshold for each subject. The EEG data were recorded and converted into the frequency domain using the fast Fourier transform (FFT) with a 4-s window and 500-ms step. During the first $4 \mathrm{~min}$, subjects were requested to stay alert and the RT of these alert trials was computed, averaged, and defined as mean RT. The averaged alpha-band power in the first $40 \mathrm{~s}$ was defined as the baseline. If a subject's response times exceeded the mean RT by 2.5 times, the subject was considered fatigued. Simultaneously, the subject's alpha-band power was recorded and averaged as the threshold, triggering a warning, and is marked as "WTH". Notably, the alpha-power threshold was set to the averaged alpha power of trials with mild fatigue (RT between 2.5 and 5 times of alert mean RT).

Fig. 1(c) presents the procedure of delivering a warning during the second session. The time interval marked as "over the warning threshold (OWT)" (Fig. 1(c)), a subject's alpha-band power exceeded WTH and the system delivered a $1,750-\mathrm{Hz}$ tone burst ${ }^{31}$ to subjects until his/her alpha-band power reduced to below WTH. However, to compare EEG signals with and without a warning, a warning was delivered in only $50 \%$ of fatigue episodes. In all experiments, the warning signal volume was set at a fixed level $(68.5 \pm 1.5 \mathrm{~dB})$, which was very noticeable but not too loud.

One might argue that if arousing feedback proves effective for improving task performance, the easiest practice of fatigue prevention seems to randomly deliver warning signals to participants. To justify the use of an EEG-based fatigue-mitigation system, ten subjects returned to participate in a control experiment in which a warning was randomly delivered to them every 15-20 min. This study then could quantitatively compare the task performance obtained by the EEG-based and nonEEG-based random fatigue mitigation systems. 


\subsection{Data analysis}

The recorded 32-channel EEG signals were first inspected to remove bad EEG channels, and then downsampled to $250 \mathrm{~Hz}$. The continuous EEG signals were segmented into 55-s epochs, from $15 \mathrm{~s}$ preceding to $40 \mathrm{~s}$ after the start of the OWT (cf. Fig. 4) or end of the OWT (cf. Fig. 6(b)). The epochs contaminated by noise signals (muscle activity, blinking, eye movement and environmental noise) were eliminated manually to minimize their influence on subsequent analysis. Notably, the channel/epoch removal procedure was only applied to offline analysis.

Independent component analysis (ICA) ${ }^{13,} 33$ was applied to decompose EEG signals into temporally independent time courses corresponding to brain and non-brain sources using EEGLAB. ${ }^{34}$ The 30-channel EEG signals were separated into 30 independent components, based on the assumption that EEG signals at sensors were linear mixtures of activation of distinct brain and non-brain sources whose time courses were statistically independent.

To identify comparable independent components across subjects, components obtained from multiple subjects were grouped into component clusters based on their scalp maps, equivalent dipole locations and baseline power spectra of component activations. ${ }^{34,35}$ Time courses of activations of components of interest were selected and transferred into the frequency domain by the Fast Fourier Transform (FFT).

The dynamic changes, tonic and phasic spectral changes of the EEG signals, were measured following the warning feedback in occipital area in this study. Tonic changes are long-time (minute-scale) variations in brain activities following a warning. Phasic changes are the short-time (second-scale) variations in brain activities in response to a warning.

The RT and EEG power were not normally distributed, such that nonparametric statistical tests were performed to analyze the data. The Wilcoxon signedrank test (Matlab statistical toolbox, Mathworks) was applied to identify significant differences among the effects of auditory feedback on RTs and changes in average EEG power spectra. Bootstrapping (EEGLAB toolbox, University of California, San Diego) was applied to determine the statistical significance of EEG power changes at specific frequency bins. To test group statistics, intrinsic inter-subject RT differences were reduced by dividing $\mathrm{RTs}$ by the mean $\mathrm{RT}$ of trials

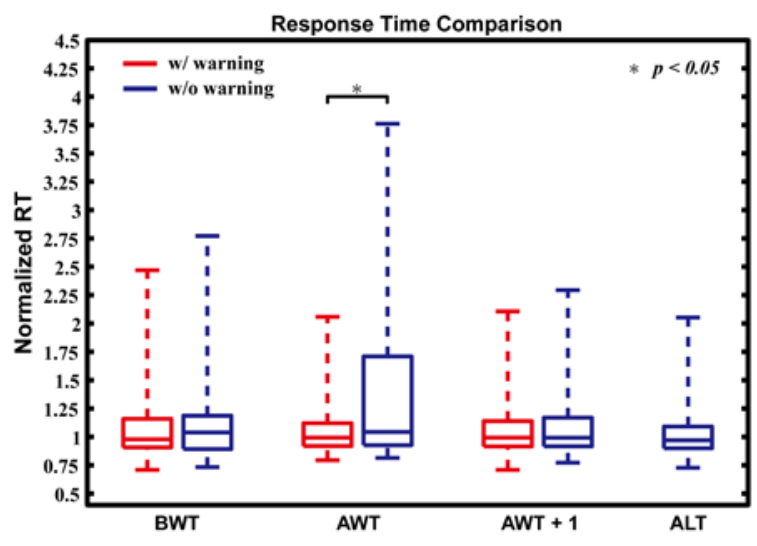

Fig. 3. Comparison of response times (RTs) of trials with warning (red boxes) and without warning (blue boxes) before and after OWT (across subjects, sessions and trials). The box plot shows the RT distributions of trials before the warning threshold (BWT), trails after the warning threshold (AWT), trials following AWT $(\mathrm{AWT}+1)$ and trials after low-alpha power (ALT) with and without warning. The middle horizontal line is the median of the distribution, and the top and bottom of the rectangle are the third and first quartile, and the dash line ends are the maximum and minimum after removing extreme values. The Wilcoxon singed rank test was applied to determine significant differences. Note: alpha-band power was under WTH for the duration of the trials before BWT.

within the first 4 min of each session. The EEG spectra were also aligned by shifting individual baseline power to all-subject average baseline power.

\section{Results}

\subsection{Comparison of behavioral performance with alpha-band power exceeding warning threshold epochs with and without a warning feedback}

Fig. 3 shows the RTs of consecutive trials before and after alpha-band power exceeded WTH. In 50\% of epochs, arousing warning signals were delivered to the subjects. Trials before the warning threshold (BWT) refer to trials with lane-departure events before a subject's alpha-band power exceeded WTH. The trials after alpha-band power declined to under WTH in $10 \mathrm{~s}$ were marked as trials after the warning threshold (AWT) and the trials following these were marked as AWT +1 . Notably, the RTs of AWT +1 were removed from analysis when a warning was delivered during the time between AWT and AWT+1. Additionally, trials after the low-alpha power (ALT) were regard as trials 
Table 1. Average RTs and standard deviations of BWT, AWT, AWT+1 and ALT (cross subjects, trials and sessions).

\begin{tabular}{cccccccc}
\hline & \multicolumn{2}{c}{ BWT } & \multicolumn{2}{c}{ AWT } & \multicolumn{2}{c}{ AWT+1 } & ALT \\
\hline & w/ warning & $\begin{array}{c}\text { w/o } \\
\text { warning }\end{array}$ & w/ warning & $\begin{array}{c}\text { w/o } \\
\text { warning }\end{array}$ & w/ warning & $\begin{array}{c}\text { w/o } \\
\text { warning }\end{array}$ & $\begin{array}{c}\text { w/o } \\
\text { warning }\end{array}$ \\
\hline Mean & 1.15 & 1.11 & 1.12 & 1.58 & 1.11 & 1.16 & 1.07 \\
\hline $\begin{array}{c}\text { Standard } \\
\text { Deviation }\end{array}$ & 0.53 & 0.38 & 0.54 & 2.31 & 0.58 & 1.10 & 0.33 \\
\hline
\end{tabular}

after the trials without a warning with a low alpha-band power (average 3-s alpha-band power after the onset of OWT was less than WTH). The RTs were normalized by dividing the respective subjects' average RTs in the first $4 \mathrm{~min}$ of the experiment during which subjects were attentive and alert (evidenced by their RTs and video recordings).

The RTs of BWT (i.e., lane-deviation events immediately before a subject's alpha power exceeded the warning threshold) in both conditions were comparable at $1 \mathrm{x}$ normalized mean alert RTs. However, the RTs of AWT with a warning (the mean RT was 1.1 times the normalized RT) were significantly shorter than those without a warning (the mean RT was 1.6 times the normalized RT) (signed-rank test, $\mathrm{p}<0.05$ ). The variation of RTs of AWT without a warning was markedly higher than that of RTs AWT with a warning. Furthermore, the RTs of BWT and AWT with a warning were comparable, suggesting that the warning effectively mitigate a fatigue-induced decrease in driving performance. In AWT+1, although the mean RTs of trials with and without a warning were comparable, the standard deviation of trials without a warning was higher than that with a warning in Table 1. Interestingly, the RTs of ALT were comparable to those of BWT under both conditions. Presumably, subjects were fairly alert in these trials and did not need any warning. The RTs of ALT immediately after low alpha power were therefore near the mean alert RT.

These empirical results demonstrate the efficacy of this warning system to rectify cognitive lapses (Fig. 3).

\subsection{Comparison of dynamic brain activities between alpha-band power exceeding threshold epochs with and without a warning feedback}

Fig. 4 shows the time courses of the fatigue-related alpha- and theta-band spectral changes in occipital components for epochs with warning (red, orange and cyan curves) and without warning (blue curve). The epochs with warning were divided into three groups according to the duration of the warning: $<3 \mathrm{~s}$ (red curve, short), 3-7 s (orange curve, medium) and $>7 \mathrm{~s}$ (cyan curve, long). All epochs were aligned to the start of OWT and transferred to the frequency domain by the FFT with a 4-s window and 200-ms step. The time courses of the alpha- and theta-band spectra were plotted from $10 \mathrm{~s}$ before and $32 \mathrm{~s}$ after the moment when the alpha-band power exceeded WTH. The red, orange and cyan horizontal dots mark the time points when the spectral difference between the trials with and without warning was statistically significant $(\mathrm{p}<0.05$, short-, medium-, long-duration warning is red, orange and blue, respectively).

For the epochs with short-duration warnings, alpha-band power increased to over $5 \mathrm{~dB}$ (from 36 to 43 $\mathrm{dB}$ ) and dropped rapidly in 1-2 s after warning onset (upper panel in Fig. 4). For the epochs with 3-7 s warning, the alpha-band power increased from 36 to 47 $\mathrm{dB}$ and peaked at $4 \mathrm{~dB}$ higher than the power with a short-duration warning. For the epochs with a longduration warning, alpha-band power increased from 36 to $45 \mathrm{~dB}$. For epochs without warning, the alpha-band power increased to $47 \mathrm{~dB}$ and then decreased to baseline slowly in $15 \mathrm{~s}$.

The lower panel of Fig. 4 shows the time courses of theta-band power. Evidently, the theta-band power trend resembled that of alpha-band power, albeit the range of fluctuations for theta-band power (36-40 dB) was smaller than that of alpha-band power. This empirical results suggest that alpha-band power fluctuations were more sensitive than theta-band fluctuations to the transition from full alertness to mild drowsiness.

The spectral difference between epochs with shortduration warnings and without warnings was 


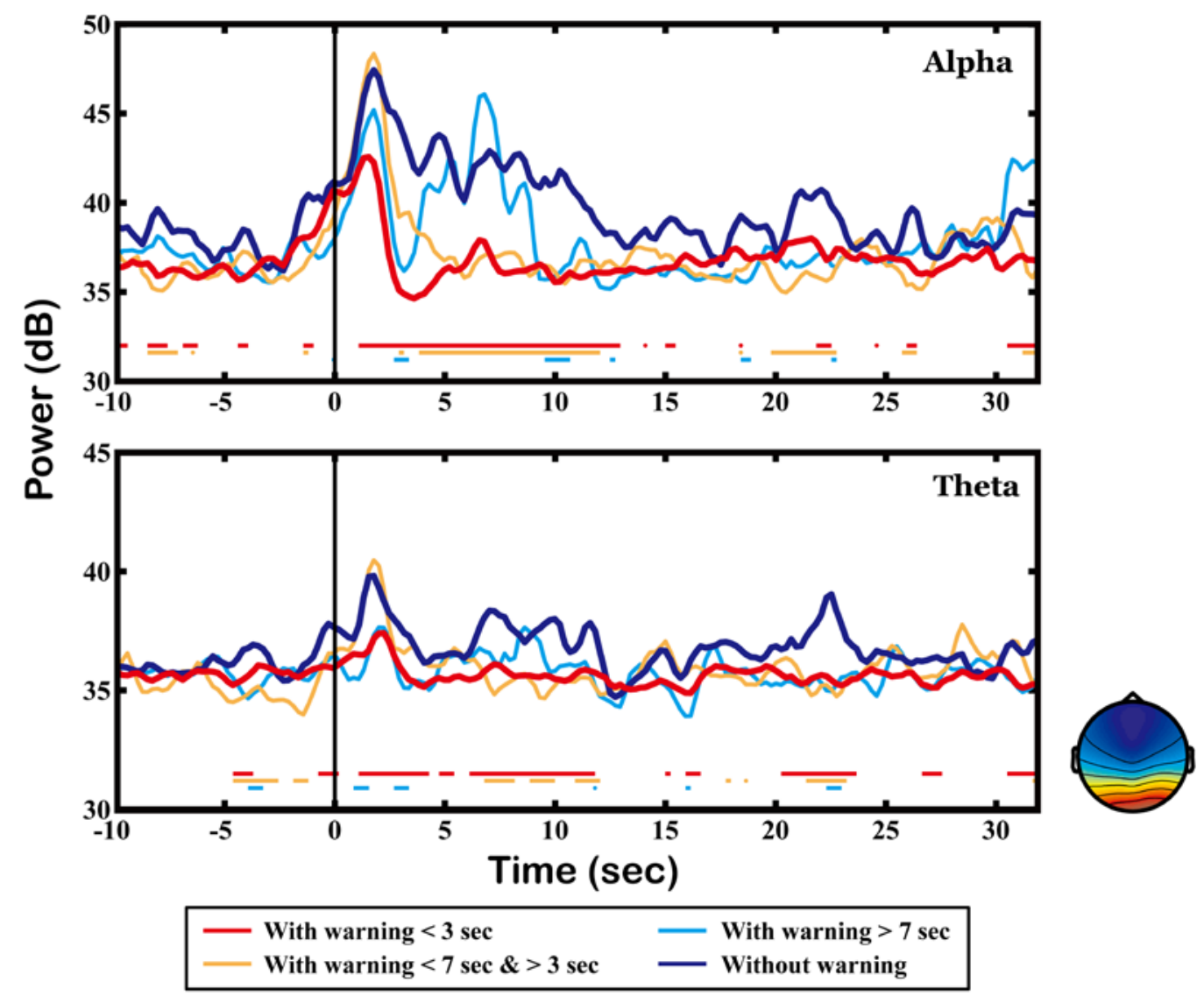

Fig. 4. Averaged (across subjects, sessions and trials) power spectral time series in alpha (upper) and theta (lower) bands of the occipital components. The spectral fluctuations were estimated using a moving discrete wavelet transform (DWT) with a 4-s time window. All trials were aligned with the starting alpha power exceeding the warning threshold (vertical black solid line). The red, cyan and orange curves are average power spectra of trials with warnings of different durations ( $<3,3-7$ and $>7$ s). Further, the blue curves are those trials without warning. The horizontal colored lines mark the spectral differences between trials with different warning durations ( $<3,3-7$ and $>7 \mathrm{~s})$ and without warning that were statistically significant (bootstrap significance test, $\mathrm{p}<0.01$ ).

statistically significant from 2-13 s after OWT in the both theta and alpha bands. Notably, once the power of epochs without warning declined to under WTH, a warning is again delivered if the power again exceeded WTH, leading to an insignificant spectral difference between a warning lasting $<3 \mathrm{~s}$ and no warning after 13 $\mathrm{s}$ in alpha and theta bands. For epochs with warning lasting 3-7 $\mathrm{s}$ and without warning, the spectral difference was statistically significant from 4-12 s in the alpha band.

Fig. 5 extends the result of Fig. 3 to divide the trials with warning into three groups according to the durations of warnings. The RTs of AWT with short- and medium-duration warnings were comparable to the mean alert RT and significantly different (signed-rank test, $\mathrm{p}<0.05$ ) from those without a warning. However, the RTs of the trials with long warnings and without warnings did not differ significantly due to the high variation of RTs and less number of trials.

\subsection{Reduced warning efficacy to the auditory feedback}

Fig. 6 shows the average alpha-band power elevations relative to the alert baseline (33.8 dB) from 5 to $20 \mathrm{~s}$ after the warning offset during different sections of the experiments. Fig. 6(a) shows the time courses of alpha power of the occipital components for epochs following an auditory warning. These time courses were selected from sessions with at least ten trials with warning. All the epochs with warning were divided into five segments based on 'sections of warning feedback' (020\%, 20-40\%, 40-60\%, 60-80\% and 80-100\%, note 


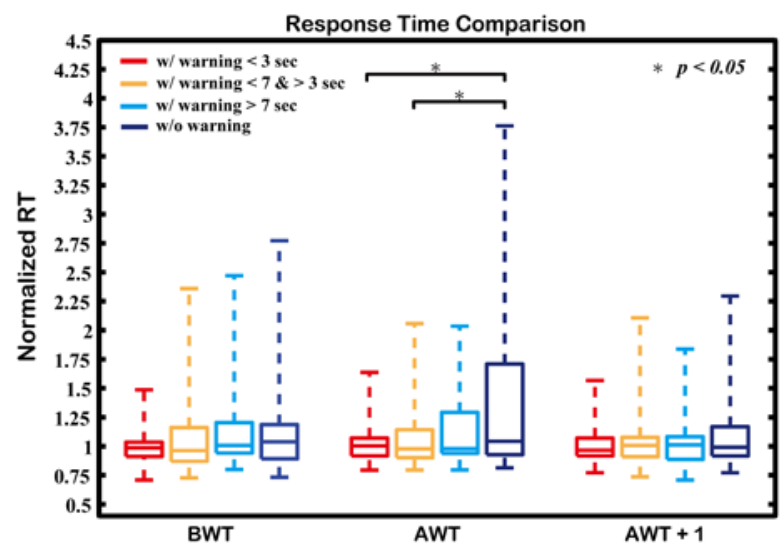

Fig. 5. Comparison of response times (RTs) of trials with warning of different durations (red, orange, and cyan boxes) and without warning (blue boxes) before and after OWT (across subjects, sessions and trials). The box plot shows the RT distributions of BWT, AWT and AWT +1 with and without warning. The middle horizontal line is the median of the distribution, and the top and bottom of the rectangle are the third and first quartile, and the dash line ends are the maximum and minimum after removing extreme values. The Wilcoxon signed rank test was applied to determine significant differences.

that the interval was left-open and right-closed) and time-aligned to the warning offset. The alpha power of the epochs in Section 40-60\% fluctuated between 36 and $43 \mathrm{~dB}$ at 5-20 s; this fluctuation was greater than that in other sections. The power fluctuation of epochs in Section $80-100 \%$ was between 36 and $40 \mathrm{~dB}$, and that in Sections $0-40 \%$ and $60-80 \%$ was between 36 and $38 \mathrm{~dB}$. Fig. 6(b) shows the alpha-band power across different sections, which was computed by averaging half-maximum power relative to the mean baseline power $(33.8 \mathrm{~dB})$ in 5-20 s after the warning offset. The power increase of $40-60 \%$ was significantly different from $0-20 \%$ and $20-40 \%$ (signed-rank test, $\mathrm{p}<0.05$ ). Additionally, the alpha power in Section $80-100 \%$ was significant higher than that in Section 20-40\% (signedrank test, $\mathrm{p}<0.05$ ).

Furthermore, Fig. 7 compares the RTs of AWT's in different sections of the experiment. The RTs in Sections $40-60 \%, \quad 80-100 \%$ and $60-80 \%$ were significantly higher than those in Sections $0-20 \%$ and $20-40 \%$ (signed-rank test, $\mathrm{p}<0.05, \mathrm{p}<0.05$ and $\mathrm{p}<0.05$, respectively). The standard deviations of RTs in Sections $40-60 \%, 80-100 \%$ and $60-80 \%$ were larger than those in Sections $0-20 \%$ and $20-40 \%$.
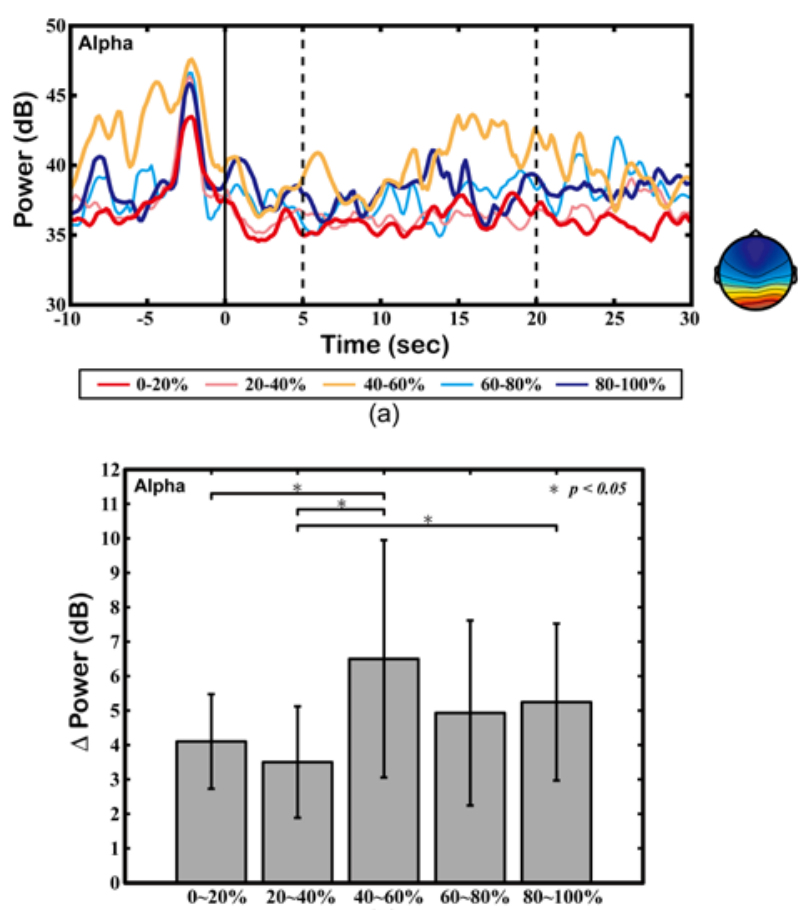

(b)

Fig. 6. Comparison of averaged occipital alpha-band power of epochs in five different sections of warning occurrence order (0-20\%, 20-40\%, 40-60\%, 60-80\% and $80-100 \%$, note that the interval was left-open and right-closed). (a) Averaged alpha-band power spectral time-series (between 10 s preceding and $30 \mathrm{~s}$ following warning offset) in different sections of the experiment. (b) The $\Delta$ Power refers to the difference between the average half-maximum alpha-band power and the mean alert baseline power $(33.8 \mathrm{~dB})$ at $5-20 \mathrm{~s}$ after warning onset. Standard deviations are also shown. The Wilcoxon signed-rank test was applied to determine significant differences $(p<0.05)$.

Both the brain dynamics and behavioral performance showed that the efficacy of warning signals often declined over time.

\subsection{Comparison of an EEG-based and a non- EEG-based random fatigue detection and mitigation system}

The analytical results obtained by this study and previous studies show that auditory feedback can mitigate the adverse effects of cognitive fatigue and alter EEG activities. ${ }^{25}$ However, they did not completely justify the necessity of EEG-based fatigue detection as one might randomly deliver arousing feedback to subjects to keep them awake. To evaluate the advantages of an EEG-based fatigue detection system over its non-EEG-based random counterpart, 10 of the 12 subjects participated in an additional simulated 


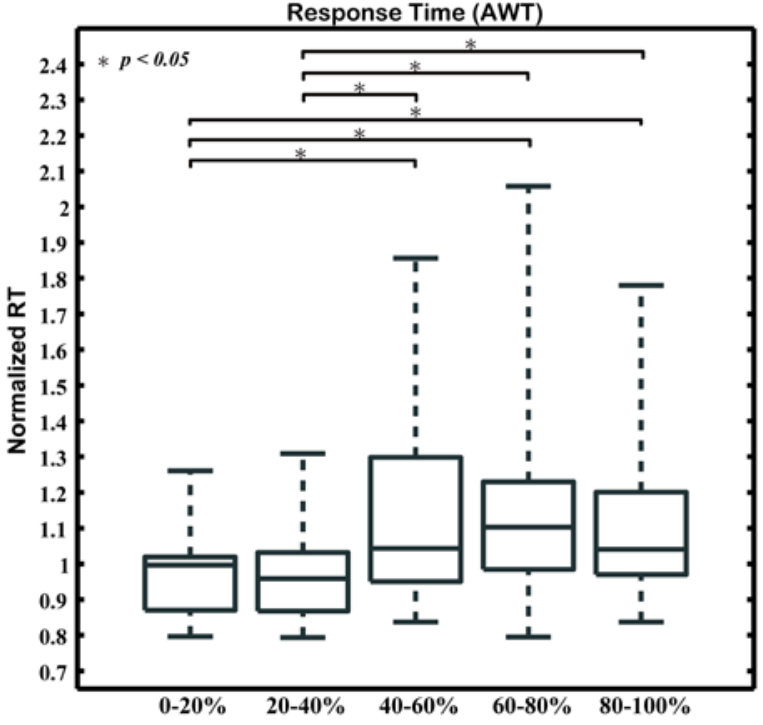

Fig. 7. The box plot of RTs of AWT in different sections (0$20 \%, 20-40 \%, 40-60 \%, 60-80 \%$ and $80-100 \%$ ) in Fig. 6. The middle horizontal line is the median of the distribution, and the top and bottom of the rectangle are the third and first quartile, and the dash line ends are the maximum and minimum after removing extreme values.

driving experiment during which a warning was delivered randomly (between 15-20 min), rather than according to subjects' EEG spectra. Fig. 8 shows 2-trial moving averaged RTs from one subject using an EEGbased fatigue detection/mitigation (red time course) vs. a non-EEG-based random method (blue time course). The EEG-based lapse mitigation system delivered a warning whenever the subject's alpha power exceeded the warning threshold. The resulting RTs never exceeded three times the mean alert RT. The RTs of the non-EEG-based random system varied widely (twice reaching 11 times the mean alert RT in Fig. 8(a)). As expected, if warning signal were delivered to the subject randomly, the system could miss periods when the subject was fatigued and non-responsive to lanedeviation events (i.e., minutes 16, 26 and 36 in Fig. 8(a)).

Furthermore, Fig. 8(b)-(j) shows the 2-trial moving average of RTs in nine additional subjects. Again, the EEG-based lapse mitigation system delivered warning signals whenever the subject's alpha power exceeded the warning threshold. The resulting RTs never exceeded three times the mean alert RT in Fig. 8(b)-(j). The RTs of the non-EEG-based random system from the subjects in Fig. 8(b)(e)(f)(g)(h) exceeded three times the mean alert RT on several occasions. These results again demonstrated that the EEG-based detection/mitigation method effectively assisted subjects in maintaining good performance during the entire experiments.

\section{Discussion}

\subsection{Changes in behavioral performance with the warning system}

Many studies, including ours, have shown brain oscillations in the alpha and theta bands were associated with fluctuations in task performance. ${ }^{13,14,25,36}$ Several studies, have also explored the use of warnings as feedback to help individuals combat drowsiness or prevent cognitive lapses. ${ }^{6-8,}$ 25, 30-32, 37-40 Our previous studies further showed that warning signals improved task performance of individuals experiencing momentary cognitive lapses and reset the EEG spectra in the alpha and theta bands to alert baseline power, ${ }^{25,32}$ suggesting that auditory feedback assisted subjects in reducing their drowsiness, reflected in both behavioral performance and brain activities.

However, the above-mentioned studies defined cognitive fatigue in terms of behavioral performance (non-responsive to lane departures). In reality, if the warning feedback is delivered to subjects after cognitive lapses, catastrophic incidents may have occurred. Thus, to be practical for real-life applications, a fatigue mitigation system needs to detect cognitive fatigue based on spontaneous EEG activities.

Wang et $\mathrm{al}^{28}$ recently proposed a smartphone-based system that detects and tracks the cognitive states of users, and delivers arousing signals that mitigate cognitive fatigue. Their study focused on the design and implementation of the fatigue detection and mitigation system and demonstrating the effectiveness of their system. However, their study did not systematically explore brain dynamics following arousal feedback. This study extended their work by exploring transient, and more importantly, tonic changes on brain activities and task performance following a warning (i.e., reduced warning efficacy). Finally, this study compared the efficacy of a closed-loop EEG-based fatigue detection and mitigation system with that of a non-EEG-based random system. The goal of this study is to investigate and justify the advantages of an EEG-based fatigue detection and mitigation system over its non-EEG-based random counterparts. 

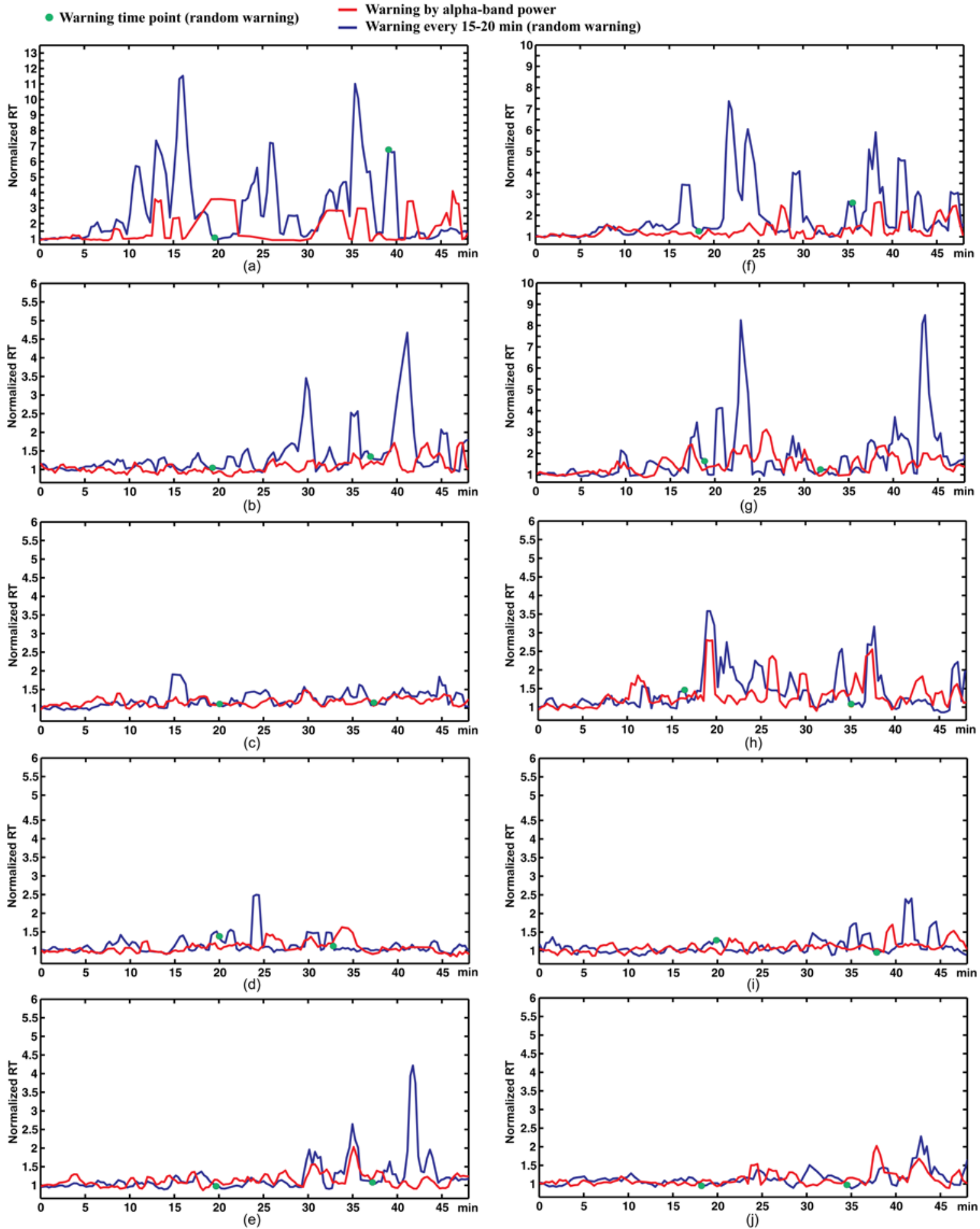

Fig. 8. A comparison of 2-trial moving averaged RTs based on two different warning strategies (EEG-based vs non-EEG-based random methods). The red curve represents RTs in the experiment using an EEG-based method, as this study designs. The blue curve represents the RTs in the experiment using a non-EEG-based random method (a random warning every 15-20 min) and the green point is the warning time (e.g. 20 and $38 \mathrm{~min}$ in (a)). The $\mathrm{x}$-axis is the processing time of the experiment. The y-axis is the normalized RT by divided the mean baseline RT. 
Additionally, it should be noted that fatigue generally accompanies drowsiness and associated with declines in attention, eventually reducing performance and efficiency ${ }^{1,2}$. Thus, both fatigue and attention might affect behavioral performance in the simulated driving task. However, Lin et al. ${ }^{25}$ and current study showed that occipital alpha- and theta-band power would increase with decreasing behavioral performance during drowsiness. In contrast, studies have shown that increasing attentional demands were associated with anterior and frontal midline theta and low alpha power. ${ }^{41}$ The current study mainly focused on the associations between the occipital theta- and alpha-band power and task performance. Study results showed increased, as opposed to decreased, theta activities in the frontal midline and occipital components, indicating subjects put forth their best attentional efforts to perform the task even during low-performance periods. These result suggested that the decline in task performance was due to fatigue.

\subsection{Effects of task performance with and without warnings}

\subsubsection{Behavioral Performance}

Our previous study ${ }^{25}$ verified the efficacy of auditory feedback by assessing the subject's RT as an index of a decrease or increase of behavioral performance. This study presented a real-time system that monitors brain dynamics and computes fluctuations of alpha-band power to identify a subject's condition. Significant changes between RTs before and after a warning were consistent with those in our previous study. ${ }^{25}$

Behavioral results in this study showed that the average RTs of epochs with warning were steady at around 1.15 times the mean alert RT. However, the RTs of trials whose alpha power exceeded the warning threshold but without receiving a warning was statistically significantly higher (and with a higher variance) (cf. Fig. 3) than those of trials following warning signals, demonstrating the necessity of the warning at that moment. Fig. 5 further splits the trials following a warning into three groups depending on the duration of the warning signals. Presumably, as a subject's fatigue increased, the mitigation system must use prolonged warning signals to arouse the subject. The mean RTs were slightly longer following longer warnings, but were still shorter than that in the OWT trials without warning (Fig. 5). These analytical results suggest that the warning feedback can effectively retain driving performance at the onset of fatigue.

\subsubsection{Brain Activities}

The EEG power spectra of the occipital component in the theta and alpha bands decreased rapidly following a warning (Fig. 4). However, the alpha-band power variation of the epochs with long warnings ( $>7 \mathrm{~s}$ ) was more fluctuant and decreased more slowly than that of epochs with short warnings (0-7 s). In addition, the power of epochs without warning also decreased slightly, which could be attributed to the fact that lane deviations aroused drivers as well (Fig. 4). Nevertheless, the alpha-band power of epochs without warning was significantly different from that of epochs with warning at 1-13 s after the OWT onset.

Fig. 4(b) shows the time courses of theta-band power, which had smaller fluctuations but the trends was comparable to that of the alpha power; power was significantly different between trials with and without warning at 1-12 s after the OWT onset. Furthermore, Fig. 4 also shows the alpha-power spectra following different durations of warnings $(<3,3-7$ and $>7$ s). The longer warning would correspond to worse fatigue level.

The spectral changes following warning feedback reported in this study were consistent with those in our previous studies, ${ }^{25}$ suggesting that auditory feedback can help subjects improve task performance.

\subsection{Reduced warning efficacy}

This study further investigated the effects of warnings over time. We hypothesize that the efficacy of a warning may decline over time due to habitation to the warning signals. Fig. 6 shows that the first $40 \%$ of warnings were most effective, evidenced by the fact that alpha power at 5-20 s after a warning offset was lower, compared to alpha power rebounds in Section 40-60\% and thereafter. Additionally, the behavioral performance in different sections also resembled the results in the alpha power (Fig. 7). According to questionnaire filled out by subjects, as many as $80 \%$ of subjects felt that a warning had less or no effect during the second half of the experiment. These findings indicate that the warnings delivered to subjects may not always be effective in arousing drowsy drivers. Future studies will explore the efficacy of changing warning signals or other modalities to mitigate cognitive fatigue. 


\subsection{Comparison of long-term behavioral changes with different warning strategies}

Fig. 8 shows the behavioral performance fluctuations in two separate 50-min lane-keeping driving experiments from ten subjects. For each subject, the red time course shows that the RTs of the session with a warning delivered based on the subject's alpha-band power were always under 3.5 times the mean alert RT (most were under 2.5 times). These analytical results were consistent with those in our previous studies. ${ }^{25}$ On the contrary, the blue time course shows that the RTs of the session with a warning delivered randomly to a subject; these RTs were often much higher and variable than those of the red time course. For example, in Fig. 8(a), half of the RTs were also over 3.5 times the mean alert RT. The random warning was delivered to the subject at around $20 \mathrm{~min}$ and $38 \mathrm{~min}$, and as expected, they resulted in short RTs in the subsequent trials. However, at 16 and $36 \mathrm{~min}$, RTs exceeded 11 times the mean alert RT (completely non-responsive) on two occasions, which may have led to devastating incidents. Fig. 8(b)(j) provides more evidence for the efficacy of the EEGbased lapse detection and mitigation system to preventing fatigue-related lapses.

\subsection{Limitations of the EEG-based fatigue detection and mitigation system}

Although the results of the current study validated the efficacy of the EEG-based system for detecting and mitigating fatigue in a driving task, it is important to keep in mind that the current demonstration still has some limitations.

First, the calibration data were needed before every online session to model subjects' physiological condition. This could be time-consuming and laborintensive and hinders the utility of BCI systems in real life. Therefore, the methods to reduce or even eliminate the amount calibration data were needed.

Second, the study findings indicate that the efficacy of warning signals delivered to drowsy subjects may decline over time, suggesting that the warning signals should be more versatile (changed over time or through other modalities) to mitigate cognitive fatigue.

Third, although the study has yielded the consistent results across the subjects participating this experiments, the more testing samples and comparison tests were still needed to confirm effectiveness and robustness of an EEG-based fatigue detection and mitigation system, and reduce possible individual physiological differences.

\section{Conclusions}

This study demonstrates the feasibility and efficacy of an on-line closed-loop fatigue detection and mitigation system based on monitoring and measuring subjects' EEG spectra noninvasively measured from the scalp. The auditory warnings can effectively arouse subjects, helping them avoid possible cognitive lapses in real environments. Furthermore, study results suggest that the efficacy of the warning signals with same modality and form may decline over time. More importantly, this study compared an EEG-based with a non-EEG-based random approach for mitigating cognitive fatigue and clearly showed the need for an adaptive fatigue detection and mitigation system that delivers warnings based on subjects' cognitive levels.

\section{Acknowledgements}

The research was supported in part by the UST-UCSD International Center of Excellence in Advanced Bioengineering sponsored by I-RiCE Program of the Ministry of Science and Technology of the Republic of China, Taiwan, under grant number: MOST 103-2911-I009-101, in part by MOST 103-2627-E-009-001, in part by the Aiming for the Top University Plan of National Chiao Tung University, the Ministry of Education, Taiwan, under grant number 101W963, and in part by the Army Research Laboratory (W911NF-10-2-0022).

\section{References}

1. R. O. Phillips, A review of definitions of fatigue - And a step towards a whole definition, Transport Res F-Traf 29 (2015) 48-56.

2. E. Grandjean, Fatigue in industry, $\mathrm{Br} J$ Ind Med 36(3) (1979) 175-86.

3. T. Balkin 2000, Effects of sleep schedules on commercial motor vehicle driver performance. (U.S. Dept. of Transportation, Federal Motor Carrier Safety Administration, Washington, DC).

4. H. P. Van Dongen, G. Maislin, J. M. Mullington and D. F. Dinges, The cumulative cost of additional wakefulness: dose-response effects on neurobehavioral functions and sleep physiology from chronic sleep restriction and total sleep deprivation, Sleep 26(2) (2003) 117-26. 
5. R. Graham, Use of auditory icons as emergency warnings: evaluation within a vehicle collision avoidance application, Ergonomics 42(9) (1999) 1233-1248.

6. C. Ho and C. Spence, Assessing the effectiveness of various auditory cues in capturing a driver's visual attention, J Exp Psychol-Appl 11(3) (2005) 157-174.

7. C. Ho, H. Z. Tang and C. Spence, Using spatial vibrotactile cues to direct visual attention in driving scenes, Transport Res F-Traf 8(6) (2005) 397-412.

8. C. Spence and C. Ho, Multisensory interface design for drivers: past, present and future, Ergonomics 51(1) (2008) 65-70.

9. P. P. Caffier, U. Erdmann and P. Ullsperger, Experimental evaluation of eye-blink parameters as a drowsiness measure, Eur J Appl Physiol 89(3-4) (2003) 319-25.

10. K. Jiao, Z. Li, M. Chen, C. Wang and S. Qi, Effect of different vibration frequencies on heart rate variability and driving fatigue in healthy drivers, Int Arch Occup Environ Health 77(3) (2004) 205-12.

11. S. Makeig and M. Inlow, Lapses in Alertness Coherence of Fluctuations in Performance and Eeg Spectrum, Electroen Clin Neuro 86(1) (1993) 23-35.

12. S. Makeig and T. P. Jung, Changes in alertness are a principal component of variance in the EEG spectrum, Neuroreport 7(1) (1995) 213-6.

13. S. Makeig and T. P. Jung, Tonic, phasic, and transient EEG correlates of auditory awareness in drowsiness, Brain Res Cogn Brain Res 4(1) (1996) 15-25.

14. T. P. Jung, S. Makeig, M. Stensmo and T. J. Sejnowski, Estimating alertness from the EEG power spectrum, IEEE Trans Biomed Eng 44(1) (1997) 60-9.

15. S. Makeig, T. P. Jung and T. J. Sejnowski, Awareness during drowsiness: dynamics and electrophysiological correlates, Can J Exp Psychol 54(4) (2000) 266-73.

16. M. A. Schier, Changes in EEG alpha power during simulated driving: a demonstration, Int J Psychophysiol 37(2) (2000) 155-62.

17. R. S. Huang, L. L. Tsai and C. J. Kuo, Selection of valid and reliable EEG features for predicting auditory and visual alertness levels, Proc Natl Sci Counc Repub China B 25(1) (2001) 17-25.

18. S. K. L. Lal and A. Craig, A critical review of the psychophysiology of driver fatigue, Biol Psychol 55(3) (2001) 173-194.

19. S. K. Lal and A. Craig, Driver fatigue: electroencephalography and psychological assessment, Psychophysiology 39(3) (2002) 313-21.

20. S. K. Lal and A. Craig, Reproducibility of the spectral components of the electroencephalogram during driver fatigue, Int J Psychophysiol 55(2) (2005) 137-43.
21. M. R. Peiris, R. D. Jones, P. R. Davidson and P. J. Bones, Detecting behavioral microsleeps from EEG power spectra, Conf Proc IEEE Eng Med Biol Soc 1 (2006) 5723-6.

22. M. T. R. Peiris, R. D. Jones, P. R. Davidson, G. J. Carroll and P. J. Bones, Frequent lapses of responsiveness during an extended visuomotor tracking task in non-sleepdeprived subjects, J Sleep Res 15(3) (2006) 291-300.

23. P. R. Davidson, R. D. Jones and M. T. R. Peiris, EEGbased lapse detection with high temporal resolution, IEEE Trans Biomed Eng 54(5) (2007) 832-839.

24. R. S. Huang, T. P. Jung, A. Delorme and S. Makeig, Tonic and phasic electroencephalographic dynamics during continuous compensatory tracking, Neuroimage 39(4) (2008) 1896-909.

25. C. T. Lin, K. C. Huang, C. F. Chao, J. A. Chen, T. W. Chiu, L. W. Ko and T. P. Jung, Tonic and phasic EEG and behavioral changes induced by arousing feedback, Neuroimage 52(2) (2010) 633-642.

26. T. P. Jung, K. C. Huang, C. H. Chuang, J. A. Chen, L. W. Ko, T. W. Chiu and C. T. Lin, Arousing feedback rectifies lapse in performance and corresponding EEG power spectrum, Conf Proc IEEE Eng Med Biol Soc 2010 (2010) 1792-5.

27. P. Artaud, S. Planque, C. Lavergne, H. Cara, P. Delepine, C. Tarriere and B. Gueguen, An on-board system for detecting lapses of alertness in car driving, Fourteenth International Technical Conference on Enhanced Safety of Vehicles, Proceedings, Vols 1 and 2 (1994) 350-359.

28. Y. T. Wang, K. C. Huang, C. S. Wei, T. Y. Huang, L. W. Ko, C. T. Lin, C. K. Cheng and T. P. Jung, Developing an EEG-based on-line closed-loop lapse detection and mitigation system, Front Neurosci 8 (2014) 321.

29. H. Wang, C. Zhang, T. W. Shi, F. W. Wang and S. J. Ma, Real-Time EEG-Based Detection of Fatigue Driving Danger for Accident Prediction, Int J Neural Syst 25(2) (2015).

30. S. M. Belz, G. S. Robinson and J. G. Casali, A new class of auditory warning signals for complex systems: Auditory icons, Hum Factors 41(4) (1999) 608-618.

31. C. T. Lin, T. T. Chiu, T. Y. Huang, C. F. Chao, W. C. Liang, S. H. Hsu and L. W. Ko, Assessing effectiveness of various auditory warning signals in maintaining drivers' attention in virtual reality-based driving environments, Percept Mot Skills 108(3) (2009) 825-35.

32. C. T. Lin, K. C. Huang, C. H. Chuang, L. W. Ko and T. P. Jung, Can arousing feedback rectify lapses in driving? Prediction from EEG power spectra, J Neural Eng 10(5) (2013) 056024.

33. A. J. Bell and T. J. Sejnowski, An informationmaximization approach to blind separation and blind deconvolution, Neural Comput 7(6) (1995) 1129-59. 
34. A. Delorme and S. Makeig, EEGLAB: an open source toolbox for analysis of single-trial EEG dynamics including independent component analysis, J Neurosci Meth 134(1) (2004) 9-21.

35. T. P. Jung, S. Makeig, M. Westerfield, J. Townsend, E. Courchesne and T. J. Sejnowski, Analysis and visualization of single-trial event-related potentials, Hum Brain Mapp 14(3) (2001) 166-85.

36. K. C. Huang, T. P. Jung, C. H. Chuang, L. W. Ko and C. T. Lin, Preventing lapse in performance using a drowsiness monitoring and management system, Conf Proc IEEE Eng Med Biol Soc 2012 (2012) 3336-9.

37. T. A. Dingus, D. V. McGehee, N. Manakkal, S. K. Jahns, C. Carney and J. M. Hankey, Human factors field evaluation of automotive headway maintenance/collision warning devices, Hum Factors 39(2) (1997) 216-29.
38. C. Spence and J. Driver, Inhibition of return following an auditory cue. The role of central reorienting events, Exp Brain Res 118(3) (1998) 352-60.

39. Y. C. Liu, Comparative study of the effects of auditory, visual and multimodality displays on drivers' performance in advanced traveller information systems, Ergonomics 44(4) (2001) 425-42.

40. J. D. Lee, D. V. McGehee, T. L. Brown and D. Marshall, Effects of adaptive cruise control and alert modality on driver performance, Transport Res Rec 1980) (2006) 4956.

41. L. I. Aftanas and S. A. Golocheikine, Human anterior and frontal midline theta and lower alpha reflect emotionally positive state and internalized attention: high-resolution EEG investigation of meditation, Neurosci Lett 310(1) (2001) 57-60. 